\title{
DEVELOPMENT OF SURVIVAL RELATIONSHIPS USING CONCOMITANT VARIABLES MEASURED FROM INDIVIDUAL SMOLT IMPLANTED WITH PIT-TAGS
}

\author{
ANNUAL REPORT \\ 1990-1991
}

Prepared by:

J.R Skalski

A. Hoffman

S.G. Smith

Center for Quantitative Science

University of Washington

Seattle, WA 98195

Prepared for:

U.S. Department of Energy

Bonneville Power Administration

Division of Fish and Wildlife

P.O. Box 3621

Portland, OR 97283-3621

Project Number 89- 107

Contract Number DE-B179-9OBPO2341

OCTOBER 1993 


\section{ABSTRACT}

The advent of PIT-tags and establishment of decoders and slide-gate facilities at hydroelectric projects on the Columbia and Snake Rivers hold the promise of providing detailed information on capture histories and travel times of individual smolt. These data. along with measurements of pre-release attributes of smolt (e.g., length, weight, condition factor, BKD-indicators, ATP-ase level, rearing history, and degree of \&scaling), traits and behavior of smolt after release (e.g., travel time, previous capture history), and ambient river conditions provide an important opportunity to determine those factors influencing survival over time and between individual smolt To this end, new statistical methods for the analysis of tag-release data are being developed concurrently with implementation of PIT-tag facilities on the Snake and Columbia Rivers.

The statistical theory and techniques of relating survival estimates from tag studies with environmental covariates is a recent development (Clobert 1985). Moreover, an ability to relate individual traits to the probable fate of individuals in a mark-recapture study was nonexistent prior to this study. The objectives of this investigation were therefore to determine the feasibility and develop new survival analyses for PIT-tag data that allowed interpretation of cohort-wise and individual covariates on smolt survival processes.

The strategy of this research has been to develop increasingly sophisticated and flexible statistical models of the analysis of PIT-tag data. The research has demonstrated not only the feasibility of incorporating cohort-wise and individual covariates in tag analysis but also the ability to simultaneously analyze multiple tag release studies and the ability to allow capture probabilities (i.e., detection probabilities) to vary as a function of an individual's release, capture history, and personal traits. 
An ongoing component of this study is the development of user-friendly software for the analysis of PIT-tag data Development of statistical software for use in an open windows computing environment on SUN workstations will permit research biologists, biometricians, and managers alike to evaluate PIT-tag data. It is anticipated that thorough analysis of survival relationships using PIT-tag data may be helpful in identifying factors influential in increasing smolt survival and suggesting adjustments for adaptive management of salmonid stocks. 


\section{INTRODUCTION}

Evaluation of the water budget, likelihood of salmon runs being listed as endangered, and continued efforts at doubling salmon stocks all focus on efforts to improve smolt survival rates. In order to enhance smolt survival during outmigration, rates of survival need to be estimated and factors influencing survival identified and altered. After years of inactivity and the advent of new technologies (e.g., PIT-tags), implementation of smolt survival studies in the Columbia and Snake Rivers appears likely in the near future. The objective of this project is to develop state-of-the-art methods for the design and analysis of smolt tag-release studies. The statistical methods developed will be based on the eventual use of PIT-tagged fish and installation of slide-gates at hydroelectric facilities.

The use of PIT-tagged fish permits recording the individual capture histories of each fish released along with measurements of their travel times over various river reaches. Coupled with this information are measurements of various traits of the individuals at the time of release. These traits may include the length, weight, condition factor, BKD indicators, ATP-ase levels, rearing history, and degree of \&scaling. Travel time is an important post-release covariate that must be evaluated All these factors are likely to contribute to the probable fate of oubnigrating smolt

The new tag-release models being developed permit survival rates to vary between individual smolt over time and allow more realistic statistical analyses of survival experiments. More importantly, by permitting survival rates to be modeled as a function of the traits and behavior of 
outmigrating smolt factors affecting survival rates can be tested and identified within a single release study. Important driving variables influencing survival can therefore be rapidly identified and modified for adaptive management of salmon and steelhead runs.

Since the advent of this BPA project, not only have nonidentically distributed survival rate models been developed but the theory has been considerably expanded Recent work has focused on the concurrent analysis of multiple-tagging studies, taking advantage of pooled sampk sixes over several releases of PIT-tagged fish. Another major development in tag-release models permits unique survival rates and unique capture probabilities for individual smolt. Allowing capture probabilities to vary between individuals permits differential detectability of fish \&pending upon release time and testing whether or not previous capture history may influence future probabilities of entering bypass units. It is anticipated these new statistical techniques will permit maximum flexibility and realism in the analysis of tag-release studies, while extracting the greatest amount of information about factors influencing survival over time and between individual smolt.

\section{METHODS}

Numerous statistical methods exist for the estimation of parameters and the analysis of tagging data (Seber 1982). The available methods for parameter estimation include maximum likelihood estimation (MLE) ordinary least squares (LS) nonlinear and weighted least squares, method of moments, and martingales. In our development of statistical methods for the analysis of PIT-tag data, we have investigated the use of MLE and martingale tbcory. 
We found MLE [for an overview of the method as applied to tag analysis, see Otis et al. (1978)] in conjunction with likelihood models composed of nonidentically but independently distributed Bernoulli trials to provide the basis for all of our survival models of PIT-tag data The statistical models of Burnham et al. (1987) are a special case of our generalized approach to PIT-tag analysis. When covariates are ignored, our likelihood models reduce to those of Burnham et aL (1987) for a single tag release under the complete capture history protocol. Moreover, the tag-release models of Burnham et al. (1987) are a special case of the Jolly-Seber model. Hence, the estimates of survival relationship we developed have a strong foundation in estimation theory and tag analysis.

In addition, we also investigated the use of martingale theory for the analysis of tagging data in survival studies. Martingale theory provides a flexible approach forỉncorporating time invariate and time varying covariates in human health epidemiological studies. In these health studies, case histories of patients can be followed in detail until the death of the patient or termination of the study. However, the incomplete observations on smolt outmigration were found to be inconsistent with the use of martingale theory. This result recommends basing the analysis of PIT-tag data on MLE methods.

\section{RESULTS}

Over the course of the study, a series of statistical models of increasing complexity and sophistication have been investigated and developed for the analysis of PIT-tag studies. In the first section, a brief description of each of the various models is given along with theii status towards user-friendly software. In the second section, a more detailed technical summary of the statistical 
notations, assumptions, and attributes of each model is provided. 'The final section includes a brief description and schematic of the statistical software being developed for the interactive analyses of PIT-tag data.

\section{Description of the Estimation M odels}

\section{Survival Only Model}

In demonstrating the feasibility of using unique survival probabilities among individuals in tag-release models, a simple likelihood model was initially developed incorporating only reach survival probablities. Capture or detection p\&abilities at each hydroelectric site were assumed to be equal to one (i.e., certainty of detection). Although not a realistic model for analysis of PIT-tag data, the model did confirm the feasibility of using survival parameters that vary between river reaches and between smolt as a function of pre-release attributes of individuals. This model is, however, applicable for the analysis of radiotelemetry data in wildlife investigations and will be submitted for publication in a suitable journal.

\section{Single Tag-Release, Pre-Release Attribute Model}

The first model developed specifically for PIT-tag studies was a single release model with reach-specific capture probabilities, site-specific baseline survival probabilities, and covariates influencing survival that vary between individuals. The current model permits a variable number 
of river reaches/decoder facilities and a variable number of covariates that can be tested for statistical significance. A computer simulation model has been developed in conjunction with the estimation algorithm to determine sample sixes for tag-release studies and to investigate robustness of those estimators to model violations. Furthermore, user-friendly software for this model is under development for SUN workstations using X-view in an Open Windows computing environment.

\section{Multiple Tag-Release, Pre-release Attribute Model}

In the future when slide-gate facilities and decoders are widely deployed, routine PIT-tag fish releases will yield repeated survival estimates over time. At that time, there will be interest in comparing the replicate survival estimates and modeling the changes as functions of ambient and smolt conditions. To this end, the single tag-release model has been extended to facilitate analysis of multiple tag-releases.

In order to analyze multipk tag-release studies simultaneous ly. the statistical models need to allow the capture process (i.e., detection rates) to change over time and locality. The multiple tag-release model developed allows the capture probabiities of individual smolt to vary as a function of cohort-wise covariates (e.g., release group, hydroelectric facility, degree of smoltification prior capture history).

A computer algorithm has been developed to estimate the parameters of this multiple tag-release model. The model permits estimation of reach-specific baseline survival rates and baseline detection probabilities at hydroelectric facilities along with regression coefficients 
asssociated with covariates describing differences in survival and detection between smolt. The next step in development is incorporating the estimation algorithm into user-friendly software for SUN workstations.

\section{Martingale Model for Post-Release Attributes}

Use of post-release covariates in smolt survival analysis introduces new technical issues in tag analysis. Principally, to unbiasedly test whether or not a covariatc may be influencing smolt survival, a subset of individuals "known to be at risk" must be identified and their fates followed. For example, to relate survival to travel time, only fish with measured travel times and known to be alive upstream can be used in the statistical analysis.

In human health epidemiological studies, martingale models provide a versatile means of incorporating pre- and post-release covariates into a proportional hazards survival model. The successful use of martingale models in human health studies suggested their potential in smolt tagging studies. However, in human health studies, the nature of censored data differs and the amount of censoring is far less than that seen in smolt tagging studies where it is essentially $100 \%$. An evaluation of martingale models applied to mark-rccaptme data showed this approach to analyzing post-release covariates to be ineffectual. Thus, we are pursuing other avenues. 


\section{Single-Release, Post-Release Attribute Model}

As an alternative to martingale theory, maximum likelihood models are being developed for the analysis of post-release covariates in tag-release studies. Maximum likelihood models have been the basis for the successful development of pre-release covariate models described above. Although the model has been expressed algebraically, it has not yet been programmed into an estimation algorithm. The post -release model will be a focus of the remainder if FY91 andFY92.

\section{Statistical Characterization of E stimation M odels}

In this section, the various survival analysis models described in the previous sections are described in greater statistical detail. The likelihood models are expressed and defined along with critical assumptions and lists of parameters that arc estimable.

Each of the following models is a function of baseline survival rate parameters and effect parameters. Together, the baseline survival and effect parameters describe the survival process within reaches. A baseline survival parameter provides a point of reference. The effect parameters then modify the baseline survival parameters for each individual according to the covariates measured. There is one effect parameter for each covariate measured so the effect on survival of each covariate can be assessed. 
As an example, consider a group of PlT-tagged chinook smolt released at the Dworshak hatchery. Before release, each smolt is weighed andmeasured. Half the smolt are fed diet A and half, diet B, prior to release. The weights and lengths are combined into a condition index weight/(length) ${ }^{3}$. Thus, each smolt has two covariates; a condition index and a diet. Thus, the model has two effect parameters, one for condition index and one for diet. Assume also that transportation does not interfere with the smolts' outmigration, so their passages are monitored at Lower Granite, Little Goose, and McNary.

One can use the statistical models we developed to test statistically whether or not the covariates affect survival. For example, if condition index has no effect on survival, then smolt with low indices are as likely to survive as smolt with high indices, and he estimate of the condition index effect parameter will be close to zero relative to its variance. If, however, there is a differential survival rate due to condition index, then the effect parameter will be far from zero relative to its variance. The sign on the parameter will describe how the effect applies, e.g., whether a low index or a high index increases survival. Similarly, one can also test if the smolt fed diet A have better chances at surviving than smolt fed diet B. Both of these analyses can be accomplished with a single tag-release study. 
Survival Only Model for Pre-Release Attributes

\section{Likelihood}

$$
L=\prod_{j=1}^{n}\left(\prod_{k=1}^{t_{j}} S_{j k}\right)\left(1-S_{j t_{j}}\right)^{I\left(t_{j}<K\right)}
$$

$\mathrm{I}(\mathrm{A})=$ indicator that event A occurred,

$\boldsymbol{t}_{\boldsymbol{j}}=$ interval in which the $j$ th individual died, $j=1, \ldots, n$,

$\boldsymbol{S}_{\boldsymbol{k}}=$ probability that the $j$ th individual survived the $k$ th reach, $\boldsymbol{j}=1, \ldots, \boldsymbol{n} ; \boldsymbol{k}=1, \ldots, \boldsymbol{K}$,

$=S_{k}^{e^{x_{j} p_{k}}}$

and where $\quad \boldsymbol{S}_{\mathbf{k}}=$ baseline probability that an individual that survived the $(\mathbf{k}-\mathbf{1}) \mathbf{t h}$ at reach survives the $k$ th reach, $\boldsymbol{k}=1, \ldots \boldsymbol{K}$,

$\boldsymbol{X}_{\boldsymbol{j}}=$ vector of covariates measured on the $j$ th individual at the time of release, $j=1, \ldots, n$,

$\boldsymbol{\beta}_{\boldsymbol{k}}=$ vector of effect parameters for the $k$ th reach, $\boldsymbol{k}=\mathbf{1}, \ldots, \mathrm{K}$. 


\section{Assumptions}

1. The fates of the individuals are independent of each other.

2. There is no tag effect on survival.

3. There is no radio-tag failure, i.e., the fate of each individual in the study is known.

4. The baseline survival rate in an interval is the same for each individual.

5. The covariates are accurately measuredon each individual.

6. The effects of the covariates on survival is the same for each individual.

\section{Capabilities}

With this model:

1. We can estimate baseline survival rates that are common to all individuals in each interval,

2. We can estimate effect parameters (i.e., $\boldsymbol{\beta}$ 's) that modify the baseline survival estimates for an individual.

3. Using the above parameter estimates, we can predict survival rates in every interval which are customized by an individual's covariates. 


\section{Likelihood}

$$
L=\prod_{j=1}^{n}\left[\prod_{k=1}^{i} S_{j k} P_{k}^{I_{j k}}\left(1-P_{k}\right)^{1-I_{j k}} \quad \xi_{j t_{j}}\right.
$$

$\boldsymbol{t}_{\boldsymbol{j}}=$ interval in which the $j$ th individual was last seen, $\boldsymbol{j}=\mathbf{1}, \ldots, \boldsymbol{n} ; \boldsymbol{t}_{\mathbf{j}}=\mathbf{0}, \mathbf{1}, \ldots, \boldsymbol{K}$, $\boldsymbol{I}_{\boldsymbol{k}}=\left\{\begin{array}{c}1 \text { if the } j \text { th individual was seen at the } k \text { th recovery site } \\ j=1, \ldots, \boldsymbol{n} ; \boldsymbol{k}=1, \ldots, \boldsymbol{K}\end{array}\right.$ $\boldsymbol{S}_{\boldsymbol{j}}=$ probability that the $j$ th individual who survived the $k$-1 st reach survived the $k$ th reach,

$$
=S_{k}^{e^{x_{j} p_{k}}}
$$

and where

$\boldsymbol{S}_{\boldsymbol{k}}=$ baseline probability that an individual survives the kth intend,

$$
\boldsymbol{k}=1, \ldots, \boldsymbol{K},
$$

$\boldsymbol{X}_{\boldsymbol{j}}=$ vector of covariates measured on the $j$ th individual at the time of release $j=1, \ldots, n$,

inctor of effect parameters for the $k$ th reach, $\boldsymbol{K}$, 
$\boldsymbol{P}_{\mathbf{k}}=$ probability that an individual who survived the $k$ th interval is recaptured at the $k$ th recoverysite, $k=1, \ldots, K$,

$\xi_{j=}=$ probability that the $j$ th individual is not seen again after the kth recovery site,

$$
=\left\{\begin{array}{l}
\theta \text { if } t_{j}=K \\
\sum_{i=t_{j}+1}^{K-1}\left\{\left(1-S_{j i}\right) \prod_{i=t_{j}+1}^{i-1} S_{j(1}\left(1-P_{l}\right)+(1-\theta) \prod_{t=t_{j}+1}^{K-1} S_{j(1}\left(1-P_{l}\right)\right\} \quad \text { if } \quad t_{j}<K
\end{array}\right.
$$

where $\quad \boldsymbol{\theta}=\boldsymbol{S}_{\boldsymbol{\pi}} \boldsymbol{P}_{\boldsymbol{K}}$ is the product of the probability that an individual survives the last interval and is recaptured at the last recovery site.

\section{Assumptions}

1. The fates of the individuals are independent of each other.

2. There is no tag effect on survival.

3. Each individual recovered can be identified, i.e., the individual tags are unique.

4. The baseline survival rate in an interval is the same for each individual.

5. The covariates are accurately measured on each individual.

6. The effects of the covariates on survival are the same foreach individual.

7. The probability of recapture at a recovery site is the same for each individual.

8. In the last interval, the probability of being seen is the same for all individuals. This includes surviving the last interval and being recaptured at the last recovery site. 


\section{Capabilities}

With this model:

1. We can estimate baseline survival rates that are common to all individuals in each interval.

2. We can estimate the effect parameters which modify the baseline survival estimates for an individual.

3. We can kst for trends in effects, e.g., does the effect of condition index on survival diminish with distance traveled?

4 - We can estimate the probability an individual will be recaptured. In the case of the Columbia River, this is an estimate of the probability a smolt will go through the bypass system.

5 . We can predict with more accuracy than the Burnham et al. (1987) model specific survival rates in every interval (except the last) because the survival rates are customized by the covariates measured on each individual. 


\section{Multiple-Release Model for Pre-Release Attributes}

\section{Likelihood}

$$
L=\prod_{l=1}^{V} \prod_{j=1}^{n_{l}}\left[\prod_{k=l}^{t_{j l}-1} S_{j k l} P_{j k l}^{I_{j k l}}\left(1-P_{j k l}\right)^{1-I_{j k l}} \xi_{j t_{j l} l}\right.
$$

$\boldsymbol{t}_{\boldsymbol{j}}=$ recovery site at which the $j$ th individual from the $l$ th release was last seen,

$$
j=1, \ldots, \mathrm{n}, ; \quad l=1, \ldots, V ; \quad t_{p}=0, \ldots, K,
$$

$\boldsymbol{I}_{\boldsymbol{j} \boldsymbol{\mu}}= \begin{cases}1 & \begin{array}{l}\text { if the } j \text { th individual from the } l \text { th release was seen at the } k \text { th recovery site } \\ \boldsymbol{j}=1, \ldots, \boldsymbol{n}_{\boldsymbol{l}} ; \boldsymbol{l}=1, \ldots, \boldsymbol{V} ; \quad \boldsymbol{k}=1, \ldots, \boldsymbol{K}\end{array} \\ \mathbf{0} & \text { otherwise }\end{cases}$

$\boldsymbol{S}_{\boldsymbol{j} \boldsymbol{\mu}}=$ probability that the $j$ th individual from the Ith release survived the $k$ th interval,

$$
=S_{k}^{e^{x_{j}^{\prime} p_{k}}}
$$

and where

$$
\begin{aligned}
\boldsymbol{S}_{\boldsymbol{k}}= & \text { baseline probability that an individual who survived the }(k-l) \text { th } \\
& \text { interval survives the } k \text { th interval, } \boldsymbol{k}=1, \ldots, \boldsymbol{K}, \\
\boldsymbol{X}_{\boldsymbol{j} \mathbf{k}}= & \text { vector of covariates measured at the time of release on the } j \text { th } \\
& \text { individual from the } l \text { th release, } \mathbf{j}=1, \ldots, \boldsymbol{n}_{\mathbf{i}} ; \quad \boldsymbol{l}=\mathbf{1}, \ldots, \boldsymbol{V},
\end{aligned}
$$


$\boldsymbol{\beta}_{\boldsymbol{u}}=$ vector of effect parameters for the lth release in the kth interval,

$$
l=1, \ldots, V ; \quad k=1, \ldots, K,
$$

$\boldsymbol{P}_{\boldsymbol{\mu}}=$ probability that the $j$ th individual from the $l$ th release who survived the $k$ th interval is recaptured at the $k$ th recovery site $, j=1, \ldots, n_{i} ; l=1, \ldots, V ; k=1, \ldots, \mathrm{K}$,

$$
=P_{k}^{e_{j}^{\prime} \eta_{t y}},
$$

and where

$$
\begin{aligned}
\boldsymbol{P}_{\mathbf{k}}= & \text { baseline probability that an individual who survived the } k \text { th } \\
& \text { interval is recaptured at the } k \text { th recovery site, } \mathrm{k}=1, \ldots, \boldsymbol{K}, \\
\boldsymbol{Y}_{\boldsymbol{j} \mathbf{k}}= & \text { vector of covariates measured at the time of release on the jth } \\
& \text { individual from the } l \text { th release that affect catchability, } \\
& \boldsymbol{j}=\mathbf{1}, \ldots, \boldsymbol{n}_{\mathbf{l}} ; \boldsymbol{l}=\mathbf{1}, \ldots, \mathrm{V},
\end{aligned}
$$$$
\boldsymbol{\gamma}_{\boldsymbol{u}}=\text { vector of effect parameters for the } l \text { th release at the } k \text { th recovery }
$$$$
\text { Site } l=1, \ldots, V ; k=1, \ldots, K,
$$

$\xi_{j+1}=$ probablity that the $j$ th individual from the $l$ th reaease is not seen again after the $k$ th recovery site,

$$
\theta \text { if } t_{z}=K
$$

$$
=\left\{\sum_{i=t_{p+1}}^{K-1}\left\{\left(1-S_{j 1}\right) \prod_{m=t_{p+1}}^{i-1} S_{j-1}\left(1-P_{j-1}\right)+(1-\theta) \prod_{m=t_{p+1}}^{K-1} S_{j-1}\left(1-P_{j-1}\right)\right\} \quad \text { if } t_{j}<K\right.
$$


where $\quad \boldsymbol{\theta}=S_{j \times 1} P_{j \mathrm{ja}}$ is the product of the probability that an individual survives the last interval and is recaptured at the last recovery site.

\section{Assumptions}

1. The fates of the individuals are independent of each other

2. The fates of the releases are independent of each other.

3. There is no tag effect on survival.

4. Each individual recovered can be identified, i.e., the individual tags are unique.

5. The baseline survival rate in an interval is the same for each individual.

6. The covaxiates are accurately measured on each individuaL

7. The effects of the covariatcs on survival arc the same for each individual within a release.

8. The probability of recapture at a recovery site is the same for each individual within a release.

9. In the last interval the probability of being seen is the same for all individuals within a release. This includes surviving the last interval and being recaptured at the last recovery site.

\section{Capabilities}

With this model:

1. We can simultaneously analyze data from multiple rcleascs. 
2. We can estimate abaseline survival rates that are common to all individuals in each interval.

3. We can estimate the effect parameters which modify the baseline survival estimates for an individual within a release. The effect parameters are common to all individuals within a release but can vary between releases.

4. We can test whether or not a covariate has the same effect on each release or whether the effect differs between releases in addition to the tests described for the single release model.

5. We can estimate the probability an individual will be recaptured. In the case of the Columbia River, this is an estimak of the probability a smolt will go through the bypass syskm.

6. As in the single-release model, we can predict with more accuracy than the Burnham et al. (1987) model specific survival rates in every interval (except the last), which are customized by the covariates measured on each individual. 


\section{Martingale Model for Pre-Release Attributes}

\section{Model}

$$
\sum_{j=1}^{n} M_{j}(K)^{2}=\sum_{j=1}^{n}\left[N_{j}(K)-\sum_{i=1}^{K} \lambda_{j}(i)\right]^{2}=0
$$

$\boldsymbol{K}=$ last recovery site

$\boldsymbol{N}_{j}(\boldsymbol{K})=$ number of time individual $j$ was seen by the time it reached recovery site $K \mathrm{j}=1, \ldots, \boldsymbol{n}$,

$\boldsymbol{\lambda}_{\boldsymbol{j}}(\boldsymbol{i})=$ probability that the jth individual is recaptured at recovery site $\boldsymbol{i}$ given the history of recaptures for and covariates measured on that individual up to recoverysite $\mathrm{i}-1 ; \mathbf{j}=1, \ldots, \boldsymbol{n} ; \mathrm{i}=1, \ldots, \boldsymbol{K}$,

$=\left[\prod_{k=L_{j(i)}}^{i-1} S_{k}^{e^{j f_{k}}}\left(1-P_{k}\right)\right] S_{i}^{e^{x j i}} P_{i}$

where $\quad \boldsymbol{L}_{\boldsymbol{j}(\boldsymbol{i})}=$ the last recoverysite the $j$ th individual was seen at by the time it arrived at the $i$ th recovery site, $j=1, \ldots, n ; i=1, \ldots, K$,

$\boldsymbol{S}_{\mathbf{k}}=$ baseline probability that an individual who survived the $k$-lst interval survives the kth interval, $k=1, \ldots, K$, 


$$
\begin{aligned}
& \boldsymbol{X}_{\boldsymbol{j}}= \text { vector of covariates measured on the } \mathrm{jth} \text { individual at the time of } \\
& \text { release } \mathrm{j}=1, \ldots, \boldsymbol{n}, \\
& \boldsymbol{\beta}_{\boldsymbol{k}}= \text { vector of effect parameters in the } k \text { th interval } \boldsymbol{k}=\mathbf{1}, \ldots, \boldsymbol{K}, \\
& \boldsymbol{P}_{\boldsymbol{k}}=\text { probability that an individual is recaptured at the } \boldsymbol{k} \text { th recovery site, } \\
& \\
& \boldsymbol{k}=1, \ldots, \boldsymbol{K} .
\end{aligned}
$$

\section{Assumptions}

1. The fates of the individuals are independent of each other.

2. There is no tag effect on survival.

3. Each individual recovered can be identified, i.e.. the individual tags are unique.

4. The baseline survival rate in a reach is the same for each individual.

5. The covariates are accurately measured on each individuaL

6. The effects of the covariates on survival are the same for each individual within a release.

7. The probability of recapture at a recovery site is the same for each individual.

8. In the last interval, the probability of being seen is the same for all individuals. This includes surviving the last interval and being recaptured at the last recovery site. 
Single-Release Model for Post-Release Attributes

\section{Comment}

This model is designed for the PIT-tag detector system currently on the Columbia River with slide gates operating at both Lower Granite and Little Goose Dams, i.e., no transportation of PIT-tagged fish. This is a first-stage model which will be generalized to more elaborate study designs.

\section{Likelihood}

The likelihood model for three river reaches can be written as:

$$
L=\prod_{j=1}^{n_{1}}\left[\prod_{k=2}^{t_{j}-1} S_{j k} P_{k}^{I_{j k}}\left(1-P_{k}\right)^{1-I_{j k}} \xi_{j t_{j}}\right.
$$

$\boldsymbol{n}_{\mathbf{1}}=$ number of individuals seen at the first recovery site,

$\boldsymbol{t}_{\boldsymbol{j}}=$ recovery site at which the $j$ th individual was last seen, $\boldsymbol{j}=\mathbf{1}, \ldots, \boldsymbol{n}_{\mathbf{1}}$,

$\boldsymbol{I}_{\boldsymbol{k}}=\left\{\begin{array}{l}1 \text { if the } j \text { th individual was recaptured at the } k \text { th recovery site, } \\ \mathrm{j}=\mathbf{1}, \ldots, \boldsymbol{n} ; \boldsymbol{k}=\mathbf{2 , 3} \\ \mathbf{0} \text { otherwise }\end{array}\right.$ $S_{j}=$ probability that the $j$ th individual survived the th interval, $j=1, \ldots, n ; k=2,3$, 


$$
=S_{k}^{e_{j} p_{k}}
$$

Where $\quad \boldsymbol{S}_{\mathbf{k}}=$ baseline probability that an individual who survived to the first recovery site survives the $k$ th interval, $\boldsymbol{k}=\mathbf{2 , 3}$, $\boldsymbol{X}_{\boldsymbol{j}}^{\prime}=$ vector of covariates measured on the $j$ th individual at the first recovery site, $j=1, \ldots, n_{1}$,

\section{$\beta_{k}=$ vector of effect parameters in the $k$ th interval, $k=2,3$,}

$\boldsymbol{P}_{\mathbf{k}}=$ probability that an individual is recaptured at the kth recovery site, $\boldsymbol{k}=\mathbf{2 , 3}$,

$\xi_{j *}=$ probability that the jth individual is not seen after the kth recovery site,

$$
\begin{aligned}
& \mathrm{j}=1, \ldots, n_{1} ; \quad k=2,3, \\
& =\left\{\begin{array}{l}
\theta \text { if } t_{j}=3 \\
\mathrm{i}-\mathrm{e} \text { if } t_{j}=2 \\
\left(1-S_{j 2}\right)+S_{j}\left(1-P_{2}\right)(1-\theta) \text { if } t_{j}=1
\end{array}\right.
\end{aligned}
$$

where $\quad \boldsymbol{\theta}=\boldsymbol{S}_{\boldsymbol{\beta}} \boldsymbol{P}_{\mathbf{3}}$ is the product of the probability that an individual survives the last interval and is recaptured at the third recovery site. 


\section{Assumptions}

1. The fates of the individuals are independent of each other.

2. There is no tag effect on survival.

3. Each individual recovered can be identified, i.e., the individual tags are unique.

4. The baseline survival rate in an interval is the same for each individual.

5. The covariates are accurately measured at the first recovery site.

6. The effects of the covariates on survival is the same for each individual.

7. The probability of recaptureat a recovery site is the same for each individual.

8. In the last inkrval, the probability of being seen is the same for all individuals. This includes surviving the last interval and being recaptured at the last recovery site.

\section{Capabilities}

With this model:

1. We can estimate the baseline survival rate in the second interval that is common to all individuals who were recaptured at the first recovery site.

2. We can estimate the effect parameters which modify the baseline survival estimates for an individual who was recaptured at the first recovery site. 
3. We can measure the effect of covariates measured after the initial release on baseline survival in the second interval, e.g., we can estimate the relationship between travel time in the Lower Granite reach on survival in Little Goose reach.

4. We can estimate the probability an individual that is recaptured at the first site will be recaptured at the second site. 


\section{Statistical Software}

Implementation of the statistical models will be based on the development of a series of user-friendly and interactive computer programs. The programs will be written for SUN workstations using X-view in an Open Windows computing environment. The statistical software being developed will be compatible with SUN workstations purchased by BPA and operate in the same computing environment as CRISP0 and CRISP1 models.

The software being developed will be capable of analyzing PIT-tag data under a variety of study designs and statistical models, simulate tag release studies for purposes of sample size

calculations and evaluation of model robustness, and provide graphical displays and summaries of captureandcovariatedata. Selectionofprogram optionswillbemenudriven (Fig. 1). The statistical analyses will provide estimates of site-specific detection probabilities, reach-specific baseline survival probabilities, and estimates of regression coefficients associated with survival covariates. In addition, the program will include an option to conduct significance tests between any two hierarchical models. The analysis will be analogous to multiple regression permitting both forward and backward deletion of covariates in modeling survival relationships. 


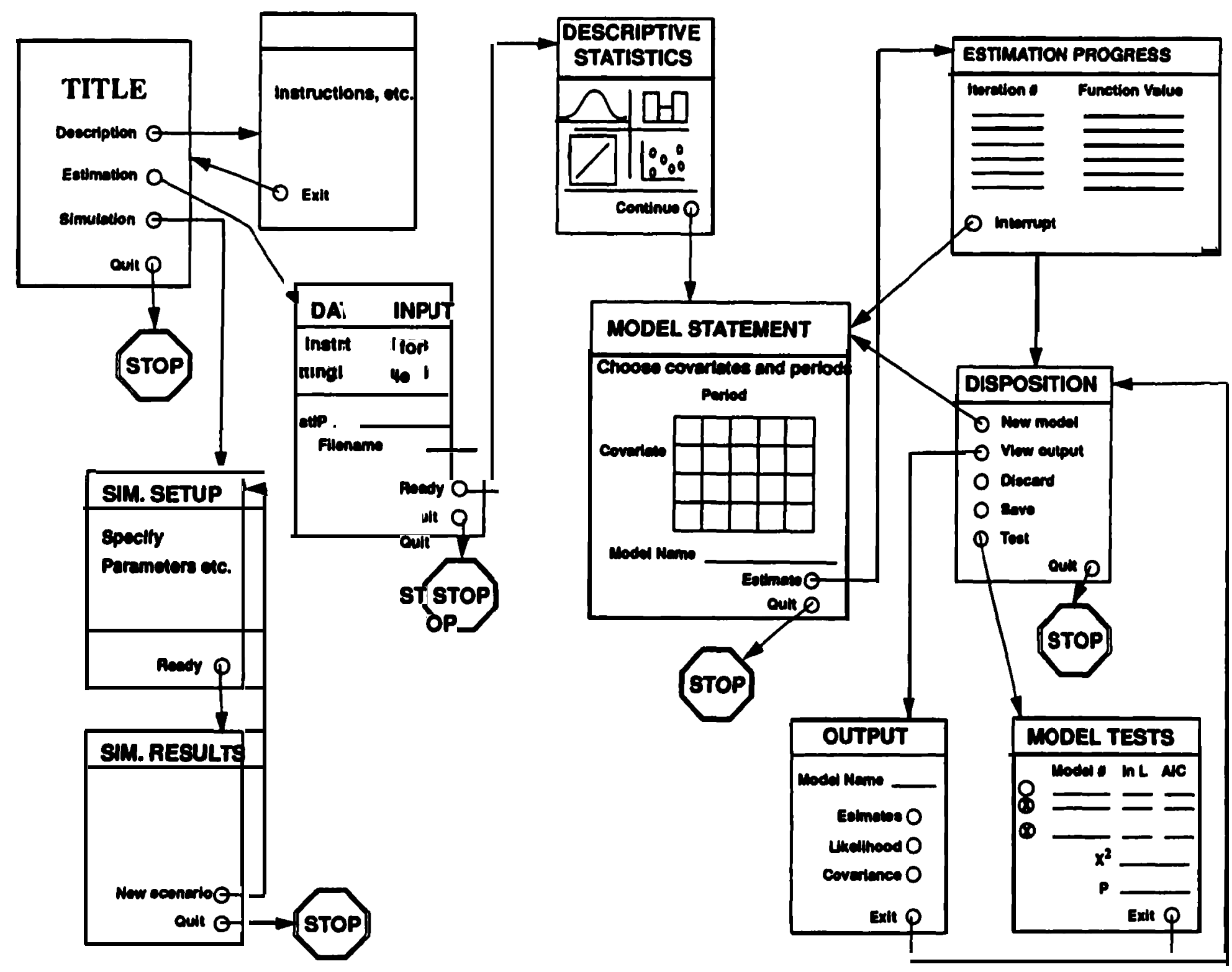

25

Figure 1. Schematic of statisticen software being $\infty$ vel pped in an Open Wineo ws environment for analysis of PIT-tag survival studies. 


\section{TIME LINE ESTABLISHED IN 1990}

A three-year time line(1990-1992) for the project was established at the onset of the program in 1990. This time line is included in the report for purposes of comparison with technical accomplishments to date and the projected time line updated as of 1991. 


\section{Time Line}

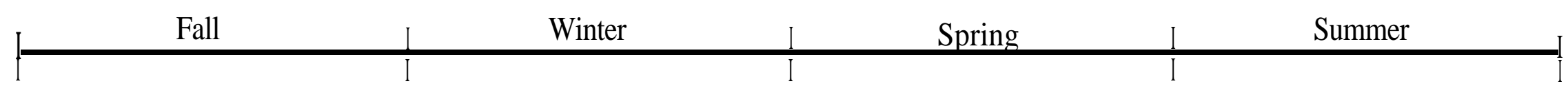

(1)Purchased computer hardware and software.

(2) Set up the computer network.
(1)Dcvelopcd the survival models.

(2) Models used prc-release covariatcs for each fish.

(3) Models are of one release and four recovery stations.

(4) Purpose: To determine the estimability of the parameters in a survival only model.
(1) Developing models with both survival terms and capture terms.

(2) Models use pre-release covariatcs for each fish.

(3) Models are of one release and four recovery stations.

(4) Purpose: To determine the estimability of the parameters in a survival and capture model.
(1) Extend the capabilities of the software to include multiple recovery stations.

(2) Models use pre-release covariates for each fish. 


\section{0-991 Time Line}

\begin{tabular}{|l|l|lll} 
Fall & Winter & Spring & I Summer \\
\hline & I &
\end{tabular}

(1) Expand the flexibility of the models to incorporate mul tiple covariatcs.

(2) Examples: Condition index, diet in hatchery, wild fish vs. hatchery fish, etc.
(1) Develop the survival models to include post-release covariates.

(2) Example: Travel time.
(1) Extend the capabilities of the post-release models to include both survival and capture terms.

(2) Models include multiple covariatcs.
Write analysis software for the models developed thus far. 


\section{Time Line}

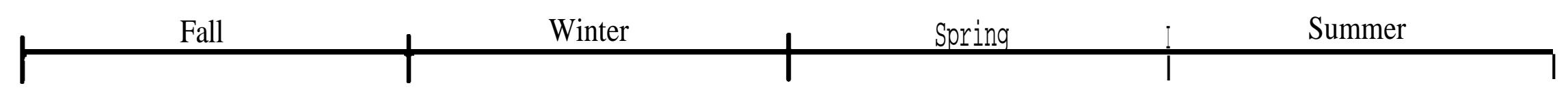

(1) Test robust properties of the models developcd.

(2) Make sample size calculations for the models.

(3) Make recommendations for PIT-tag study designs. 


\section{TIME LINE ESTABLISHED IN 1991}

An updated time line for the project as envisioned in May 1991 is included below. Comparison of the projected accomplishments (i.e., time line established in 1990) for 1990 and 1991 with the rcalizcl accomplishments (i.e., time line cstablished in 1991) for those years agree very welL The agreement between time lines affirms the project is on time and results accomplished as expected.

One deviation is in the development of a post-release covariate model. The failure of martingale theory to adequately describe tag-release data has set back the development of a post-release covariate model approximately six months. Current work on maximum likelihood models will be used to fulfill that project deliverable. Alternatively, the multiple-release, pre-release attribute model was not originally proposed but was developed within the last year because of a need identifii that was not previously perceived. The developments of the multiple-release model offsets the work on the post-release model still to be developed. It is anticipated the post-release model will be successfully completed by the end of the project. 


\section{Time Line}

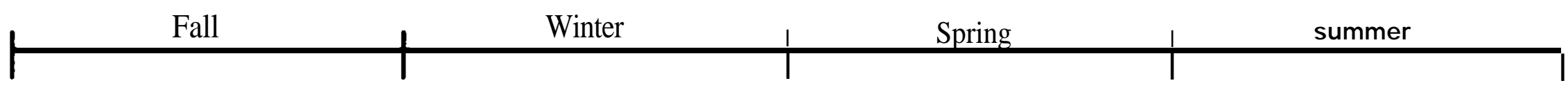

(1) Purchascd computer hardware and software.

\section{(2) Set upthecomputer network.}

(1) Developed the survival models.

(2) Models used pre-release covariatcs for each fish.

(3) Models are of one release and four recovery stations.

(4) Purpose: To determine the estimability of the parametersin a survival only model.
(1) Developed models with both survival terms and capture terms.

(2) Models used pre-release covariatcs for each fish.

(3) Models are of one release and four recovery stations.

(4) Purpose: To determine the estimability of the parameters in a survival and capture model.
(1) Extended the capabilities of the software to include multiple recovery stations.

(2) Models used pre-release covariates for each fish. 


\section{0-991 Time Line}

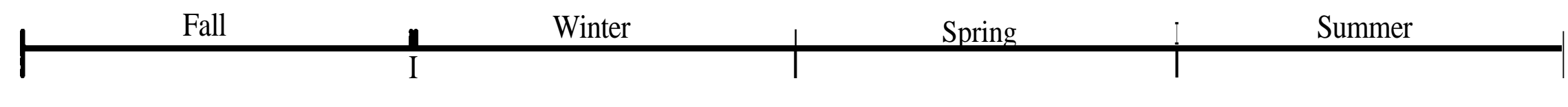

(1) Expanded the flexibility of the models to incorporate multiple covariates.

\section{Examples: condition} in\&x, diet in hatchery, wild fish vs. hatchery fish, etc.

(2) Developed martingale model for post-release covariates.

Example: travel time.
(1) Tested martingale model forpost-releasecovariates.

Result: it performed poorly.

(2) Did preliminary robustness studies to violations of assumptions in pm-release covatiate model and presented results at the Pacific Fishery Biologists meeting.
(3) Began writing user-friendly software for pre-release covariate model.

(1) Developing another post-release model based on multinomial likelihood.

(2) Continuing robutsess studies on violations of assumptions in pre-release covariate model.

(3) Beginning robustness studies on violations of assumptions in the Burnham model in conjunction with Al Giorgi.

(4) Continuing writing user-friendlysoftware for pre-release covariate model.
(1) Program the second post-release model and test performance

(2) continue writing user-friendly software for pm-release covariate model.

(3) Begin writing user-friendly software for post-release covariate model. 


\section{1-1992 Time Line}

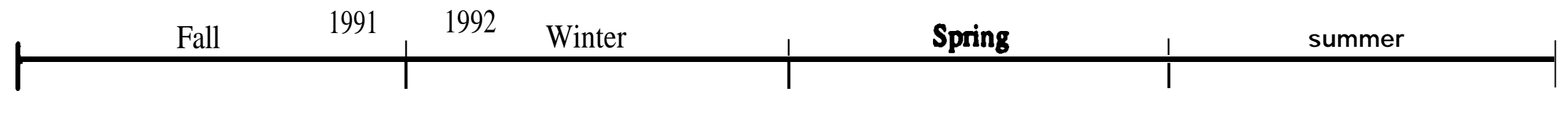
(1) Do robustness studies on
post-release covariate model.

(2) Finish writing user-friendly software for pre-release covariate model.

(3) Continue writing user-friendly software for post-release covariate model.
(1) Do sample size calculations to be used in spring PIT-tag release programs.

(2) Make recommendations for study designs for spring PIT-tag release programs

(3) Finish writing user-friendly software for post-release covariate model.
(1) Analyze PIT-tag data fromreleases.

(2) Form recommendations for future PIT-tag study designs.
(1) Hold workshops to demonstrate use of the software.

(2) Write and refine a user's manual. 


\section{LITERATURE CITED}

Bumham, K. P., D. R Anderson, G. C. White, C. Brownie, and K. H. Pollack. 1987. Design and analyses methods for fish survival experiments based on release-recapture. Amer. Fish. Soc. Monog. No. 5. 437 pp.

Clobert, J., J. D. Lebreton, M. Clobert-Gillet, and H. Coquillart. 1985. The estimation of survival in bii populations by recapture or rcsighting of marked individuals. In: B. J. T. Morgan and P. M. North (eds.), Statistics in Ornithology, New York, Springer-Verlag, pp. 197-213.

Otis, D. L., K. P. Burnham, G. C. White, and D. R Anderson. 1978. statistical inference from capture dam on closed animal populations. Wildl. Monog. No. 62135 pp.

Seber, G. A. F. 1982. The estimation of animal abundance and related parameters. MacMillan, New York 654 pp. 